Article

\title{
Assessing entomological and epidemiological efficacy of pyriproxyfen- treated ovitraps using saliva samples
}

\author{
Antonio D. Ligsay ${ }^{1,2,}{ }^{*}$, Kristan Jela M. Tambio ${ }^{2}$, Michelle Joyce M. Aytona ${ }^{2}$, Grecebio Jonathan D. Alejandro ${ }^{1}$, Zypher Jude \\ G. Regencia ${ }^{3,4}$, Emmanuel S. Baja ${ }^{3,4}$ and Richard Edward L. Paul ${ }^{5}$ \\ ${ }^{1}$ The Graduate School \& College of Science, University of Santo Tomas España Blvd., \\ Manila 1008 Philippines; gdalejandro@ust.edu.ph (G.J.D.A.) \\ ${ }^{2}$ Clinical Research Section, St. Luke's College of Medicine - William H. Quasha \\ Memorial, 279 E. Rodriguez Sr. Ave, Quezon City, 1112 Philippines; \\ kristan.tambio@gmail.com (K.J.M.T.); mjaytona@gmail.com (M.J.M.A.) \\ ${ }^{3}$ Department of Clinical Epidemiology, College of Medicine, University of the \\ Philippines Manila, Pedro Gil Street, Ermita, Manila, 1000 Philippines; \\ zgregencia@up.edu.ph (Z.J.G.R.); \\ ${ }^{4}$ Institute of Clinical Epidemiology, National Institutes of Health, University of the \\ Philippines Manila, 623 Pedro Gil Street, Ermita, Manila, 1000 Philippines; \\ esbaja@up.edu.ph (E.S.B.) \\ ${ }^{5}$ Institut Pasteur, Functional Genetics of Infectious Diseases Unit, UMR 2000 (CNRS), Paris \\ 75015 France; richard.paul@pasteur.fr (R.E.L.P.) \\ *Correspondence: adligsay@ust.edu.ph (A.D.L.)
}

\begin{abstract}
Our study assessed the impact of using ovitraps with pyriproxyfen on mosquito populations and the feasibility of using human saliva samples to test for seroconversion to dengue virus (DENV). We used a quasi-experimental research design by forming the intervention $(n=220)$ and the control $(n=223)$ groups in neighboring Taguig City, Philippines, over four months. Socio-demographic data, entomological indices, and IgG antibodies against DENV were measured. Associations between the implementation of ovitraps dosed with pyriproxyfen and mosquito densities (percentage positive ovitraps and container indices) and DENV seroconversion were calculated post-intervention in Months 2, 3, and 4. Among participants recruited at baseline, 17 and 13 were seropositive for dengue (DENV) in the intervention and control groups, respectively. Both entomological indices were lower in the treated area than the control site at months two, three, and four post-interventions but not earlier. Dengue seroconversions rates did decrease in the treated population but not significantly so. In conclusion, the use of PPF-treated ovitraps impacted the mosquito population but not the seroconversion rates. Compliance to provide saliva samples and the ability to detect IgG antibodies within these samples were encouraging and suggested that further studies on a larger population and longer duration are warranted.
\end{abstract}

Keywords: Mosquito control, Dengue, Mosquito density, Pyriproxyfen, Philippines

1. Introduction

Over the last five decades, the incidence of dengue has increased more than 30-fold, causing an estimated 50-100 million cases annually that are spread across more than 100 
endemic countries [1]. This substantial increase in the burden of dengue is considered to have been exacerbated by increased international transport and travel that has facilitated the global spread of the mosquito vector spp. and the virus [2]. Moreover, insofar as the significant tropical vector, Aedes aegypti, is well adapted to the urban environment, the increasing levels of urbanization are exposing an ever-increasing population to dengue virus (DENV) [3-5]. The public health significance of urban mosquito-borne disease is not only restricted to dengue, as evidenced by the recent outbreaks of other arboviruses, notably Zika and chikungunya, along with the continuing burden of urban malaria in India by Anopheles stephensi, which is likewise adapted to an urban environment [6-11]. The threat from arboviruses in urban settings is no longer restricted to the tropics and subtropics, as many invasive mosquito vectors can survive in temperate climates [12-14]. Outbreaks of many arboviral diseases, including dengue, chikungunya, and West Nile, have occurred in Europe and the US [15-17]. In addition, global climate warming is projected to expand the range of significant mosquito vectors into new areas, extend the transmission season in current endemic settings, and increase the mosquito's vectorial capacity $[18,19]$. Urban mosquito-borne diseases are thus projected to impose an ever-increasing public health burden on society and require urgent attention [14]. In the absence of buyin for potentially effective vaccines, reducing human contact with vectors is the only way to reduce the burden of dengue and other mosquito-borne diseases [20].

Current mosquito control approaches include the use of larvicides in stored water containers to eliminate the juvenile stages of the mosquitoes, adulticides to kill adult stages of the mosquito, and source reduction by improved environmental hygiene (eliminating solid waste that offers potential aquatic habitats for larvae). Adulticides are generally employed in and around houses of clinical cases identified through the public health system and provide short-term control. In contrast, source reduction through eliminating oviposition sites before the rainy season has shown some success but requires enormous effort [21]. Insect Growth Regulator mimics such as Novularon, Methoprene, and Pyriproxyfen (PPF) have been used extensively in mosquito control programs worldwide (22-26). Granular or liquid formulations of PPF applied to water containers and drains in South America, and Southeast Asia have had documented success in reducing immature and adult mosquito numbers and have been associated with a reduction in dengue incidence $(22,27)$. Furthermore, PPF prevents larval and pupal development and impacts female fertility and male spermiogenesis $(28,29)$.

In response to the growing global threat imposed by mosquito-borne diseases and recognizing that current methods are inadequate, the World Health Organization launched its 2017-2030 Global Vector Control Response to promote the development of novel approaches that show not only entomological but also epidemiological efficacy [22].

Successful measurement of epidemiological efficacy is challenging. A substantial but variable proportion of DENV infections are subclinical. Thus the sample size required to detect an effect of mosquito control through a measure of disease incidence would need to be very large (31). Seroconversion rates provide a more accurate measure, but the spatial and temporal unpredictable heterogeneity in dengue epidemiology means that repeated sampling from individuals is needed to generate an interpretable result. Repeated blood sampling is problematic because of compliance. Recently, saliva samples have been proposed to detect antibodies against DENV and offer a non-invasive alternative to blood sampling (32).

Here we present a study in Taguig City, Philippines, to assess the potential for this approach to show epidemiological efficacy and determine the period necessary to observe any entomological impact of using ovitraps laced with PPF.

\section{Materials and Methods}




\subsection{Study Design, Sites, and Population}

Our study used a quasi-experimental research design composed of two groups, with and without intervention. Data on dengue incidence were obtained from the Department of Health (DOH) - Metro Manila Centers for Health Development. Cities in the National Capital Region with the highest dengue attack rate were candidate areas for the study. Taguig City, a densely populated area in the south of Manila, was chosen. Criteria for selecting sites within Taguig included small municipalities/barangays constantly reporting 10-50 dengue cases monthly. The barangay of Western Bicutan was chosen, housing approximately 100,000 individuals. The Katipunan area was selected as the control site and the Philippines National Railways area as the intervention site. These sites are very nearby but not juxtaposed. Intervention and control sites were matched as closely as possible according to the history of local dengue incidence. Once a dengue index case was signaled within the sites, ovitraps (mosquito density monitoring devices) were implemented for two weeks to assess whether mosquito densities ( $>5 \%$ ovitraps positive for mosquito larvae) were sufficiently high for active DENV transmission and therefore selectable as a study site. Figures $1 \mathrm{a}$ and $1 \mathrm{~b}$ show the schematic of the two study sites with a distribution of ovitraps to measure suitability for study once a dengue index case was detected. 


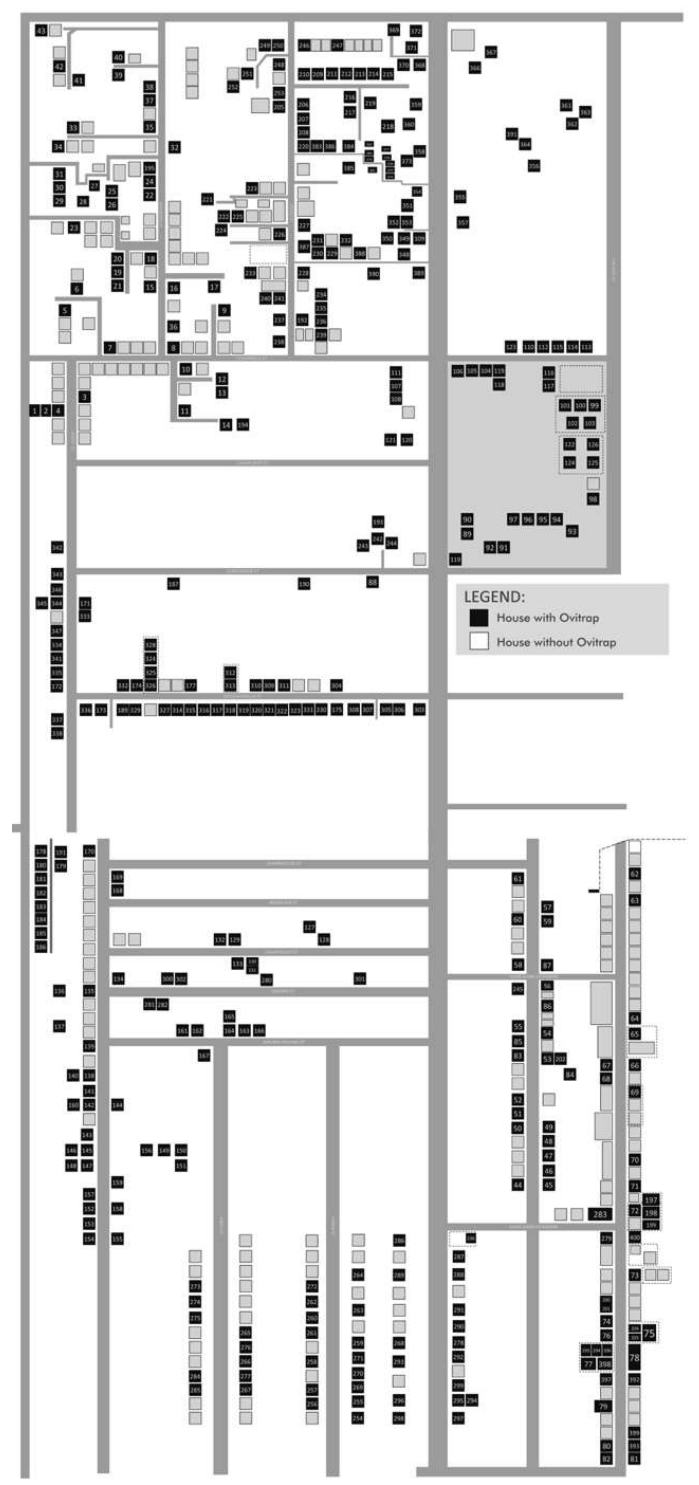

Figure 1a. Schematic Trial Design for the Control Area (Katipunan Area). 


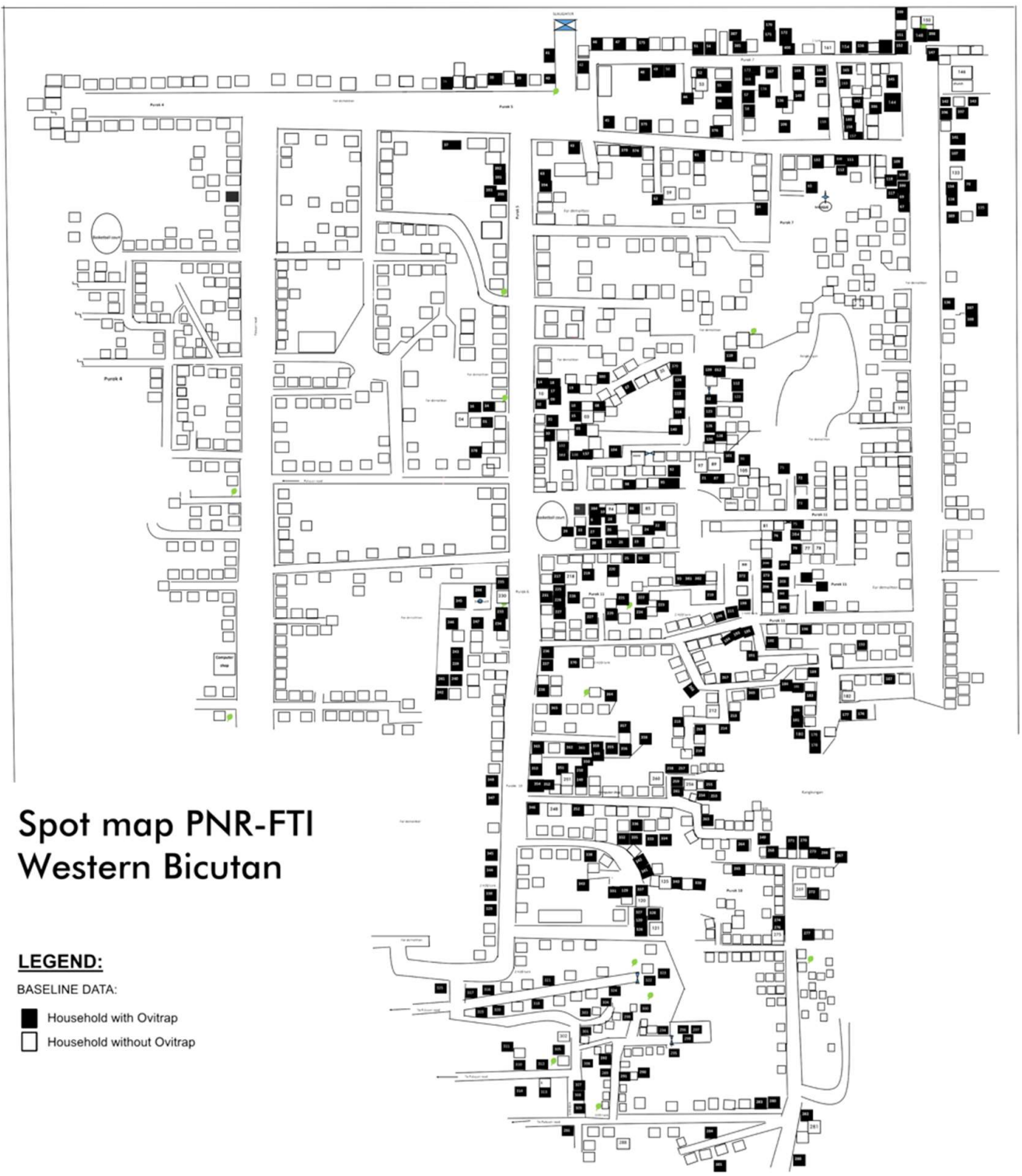

Figure 1b. Schematic Trial Design for the Intervention Area (PNR Area).

\subsection{Inclusion and Exclusion Criteria of Participants}

\subsubsection{Inclusion Criteria}

After selecting the site, we invited participants living within 100 meters of the dengue index case. Our target study population was 200 per intervention site. Invited participants were individuals aged 1-30 years old who were willing to participate in the study and could provide saliva samples for DENV antibody detection. A baseline saliva sample was taken at the time of recruitment, kept on ice before storage at $-80^{\circ} \mathrm{C}$ awaiting analysis. 2.2.2. Exclusion Criteria

At the time of collection, individuals who were febrile, suffering from any chronic disease, and refused to sign the informed consent were excluded. 


\subsection{Data Collection}

\subsubsection{Entomological indices, Intervention, and Control Ovitap Set-Up}

Following the parameters set by the WHO, different entomological indices were measured. We used the Container index (CI - the percentage of water-holding containers infested with larvae and/or pupae) and the Ovitrap Index (OI - Percentage of positive ovitraps, larvae, and/or pupae) [23]. Ovi-PPF devices (ovitraps filled with $250 \mathrm{ml}$ of water with one sachet of Sumilarv ${ }^{\mathrm{TM}}$ 0.5G Sumitomo Chemical) were placed in the selected households in the intervention study site houses at the time of recruitment. In the control site, ovitraps without PPF solution were similarly deployed. One trap was placed per house indoors. Ovitraps were inspected weekly by the barangay health workers, and the contents were serviced every two weeks. Should the contents be less than the required volume (half full), the ovitrap (with or without PPF) was refilled. Container indices were calculated on a weekly basis by inspecting the number of water-filled containers positive for mosquito larvae/pupae. Water-field containers found positive for larvae were then emptied and if serving as water storage were then re-filled.

Participating houses that were directly adjacent were combined into clusters, and mosquito indices were calculated at the cluster level. If there were no containers containing water in a cluster at a given time, the CI was marked as missing data but not zero. Weekly cluster level indices were averaged over the month.

\subsubsection{Outcome Assessment: Antibody Measurements}

A saliva sample for baseline IgG determination was carried out among participating individuals in the intervention and control groups at month 0 . At that time, information on the age and gender of participants, household income, and level of education of parents were ascertained. Individuals were anonymized with codes assigned. Ovitraps with PPF were then deployed. The first post-intervention saliva monitoring was done after two months and then monthly for a total of three monitoring visits. Thus, the Ovi-PPF traps were in place for a total of four months.

The IgG ELISA test was done according to the procedures previously described [24]. Positive and negative controls previously confirmed as control references were provided by Institut Pasteur Cambodia [25]. Samples were analyzed in duplicates. In case there were conflicting results, a third test was performed. An optical density (OD) difference between the sample and the control of 0.1 and above was considered positive for IgG. Only those participants who were IgG negative during the baseline sample were included in the subsequent analysis. Likewise, individuals seroconverting later (month two and month three) were removed from further sampling and analysis.

\subsection{Data Analysis}

Descriptive statistics for the baseline study characteristics of the participants were calculated. In addition, Ovitrap and Container Indices were first tested for normality using the Shapiro-Wilk test. Since being not normally distributed, these mosquito indices were arcsine transformed. The association of site type (control vs. treatment) with mosquito indices were analyzed by fitting a Generalized Linear Model with a normal distribution, identity link function, and the significance assessed through F statistics [26].

For the baseline seropositive analysis, bivariate analyses were performed. Moreover, the following explanatory variables were considered, including age (child, $1-12$ years old; adolescent, 13 - 18 years old; young adult, 19 -30), sex (male; female), father's education (no education to high school graduate; college to graduate school), mother's education (no education to high school graduate; college to graduate school), and family income (no income to < PHP 15,000.00; PHP 15,000.00 and above) and site type. Generalized Linear Models were fitted to estimate the effect of every explanatory variable on seropositivity at baseline. 
Seroconversion data in months two, three, and four post-interventions (or control) were analyzed by fitting a Generalized Linear Mixed Model (GLMM) with a binomial distribution and logit function. Moreover, bivariate and multivariable analyses were performed. In the multivariable model, these explanatory variables were considered: Age (child, 1 - 12 years old; adolescent, 13 - 18 years old; young adult, 19 - 30), sex (male; female), father's education (no education to high school graduate; college to graduate school), mother's education (no education to high school graduate; college to graduate school), family income (no income to < PHP 15,000.00; PHP 15,000.00 and above), site type, month, the arcsine transformed mosquito indices and the interaction between site type and month. House number was fitted as the random term.

In addition, IgG optical density, not being normally distributed, was analyzed by Poisson regression with individual ID fitted as the random term in the GLMM [27]. In all cases, a dispersion parameter was estimated to account for any over-dispersion in the data. Finally, overall significance was assessed by calculating Wald statistics, and stratum level differences (i.e., between levels within a factor) were calculated through t-tests. Analyses were performed in Genstat Vers. 20.

\subsection{Ethical Considerations}

Ethics approval was sought from an Independent Research Ethics Committee. The study was conducted in compliance with the procedures outlined in this protocol and the National Ethical Guidelines for Health Research 2011 following the Data Privacy Act of 2012.

\section{Results}

The study recruited 443 participants, of which 220 belonged to the intervention group and 223 belonged to the control group. These individuals were spatially distributed into 41 control area clusters and 48 treatment area clusters, where a cluster is defined as having participating individuals in directly adjacent hoses. The number of houses per cluster ranged from one to ten, median four and there was a total of 327 participating houses. The number of participating individuals per house ranged from one to five, with $76.5 \%$ of houses having a single participating individual. As the study progressed and participants who seroconverted were subsequently excluded from future analyses, the total number of participants and houses decreased, reaching 281 houses in 41 control and 46 treatment clusters and 357 individuals by the final month of the study.

At baseline, 17 and 13 individuals were $\operatorname{IgG}$ positive in the intervention and control groups, respectively. These seropositive individuals were distributed across 11 houses in 7 clusters in the control site and 17 houses in 10 clusters in the intervention site. Of the 11 control site houses with a seropositive individual, five houses had more than one person tested, of which none had more than one seropositive individual. Of the 17 positive treatment site houses, seven had more than one tested individual, and only one house had more than one person seropositive.

Table 1 gives the baseline characteristics of the participants. The intervention and control groups had an average age $( \pm$ SD) of $14.6( \pm 7.1)$ years old and $13.9( \pm 8.4)$ years old, respectively $(p$-value $=0.39)$. Females comprised a similar and higher number of participants in both the intervention $(53 \%)$ and control $(54 \%)$ groups ( $p$-value $=0.89)$. The intervention group's average monthly household income $( \pm \mathrm{SD})$ was Philippine Pesos (PHP) $12,773 \pm 13,465$ compared to PhP 12,070 \pm 7,467 in the control group. In addition, the categorization of the household income revealed that most of the participants in both the intervention (73\%) and control group (55\%) fell under the PHP 10,001 to < 20,000 income bracket. Further analysis showed that overall, the average monthly household income for the participants did differ significantly between intervention and control groups ( $p$-value $<0.01$ ) (Table 1). The average baseline IgG ODs for each of the groups were not 
significantly different $(p$-value $=0.55): 0.046( \pm 0.036) \mathrm{g} / \mathrm{L}$ and $0.044( \pm 0.033) \mathrm{g} / \mathrm{L}$ for the intervention and control groups, respectively.

In the univariate statistical analyses, there was no association of seropositivity at baseline with sex $\left(\chi^{2}=0.08, p\right.$-value $\left.=0.371\right)$, age category $\left(\chi^{2}=0.25, p\right.$-value $\left.=0.881\right)$, Income category $\left(\chi^{2}=2.33, p\right.$-value $\left.=0.313\right)$, Mother's education $\left(\chi_{1}^{2}=0.08, p\right.$-value $\left.=0.773\right)$, Father's education $\left(\chi^{2}{ }_{1}=0.01, p\right.$-value $\left.=0.941\right)$ or site type $\left(\chi^{2}{ }_{1}=0.52, p\right.$-value $\left.=0.571\right)$. No multivariable analyses were subsequently carried out as no variables had a $p$-value $<0.25$ in the univariate analysis.

Table 1. Baseline characteristics of the study population, by the site $(\mathrm{N}=443)$.

\begin{tabular}{|c|c|c|c|c|c|c|}
\hline \multirow{2}{*}{ Characteristics } & \multicolumn{2}{|c|}{ Intervention Group } & \multicolumn{2}{|c|}{ Control Group } & \multicolumn{2}{|c|}{ Total } \\
\hline & $\mathbf{n}$ & $\%$ & $\mathbf{n}$ & $\%$ & $\mathbf{n}$ & $\%$ \\
\hline \multicolumn{7}{|l|}{ Age, years old } \\
\hline Child, $1-12$ & 99 & 45 & 73 & 32.8 & 172 & 38.83 \\
\hline Adolescent, 13 - 18 & 60 & 27.3 & 38 & 17 & 98 & 22.12 \\
\hline Young adult, $19-30$ & 61 & 27.7 & 112 & 50.2 & 173 & 39.05 \\
\hline \multicolumn{7}{|l|}{ Sex } \\
\hline Male & 103 & 46.8 & 103 & 46.2 & 206 & 46.50 \\
\hline Female & 117 & 53.2 & 120 & 53.8 & 237 & 53.50 \\
\hline \multicolumn{7}{|l|}{ Income, $\mathrm{PhP}$} \\
\hline No income & 3 & 1.4 & 19 & 8.5 & 22 & 4.97 \\
\hline 1 to $<10,000$ & 41 & 18.6 & 48 & 21.5 & 89 & 20.09 \\
\hline 10,001 to $<20,000$ & 161 & 73.2 & 123 & 55.2 & 284 & 64.11 \\
\hline 20,001 to $<30,000$ & 14 & 6.4 & 24 & 10.8 & 38 & 8.58 \\
\hline 30,001 and above & 1 & 0.4 & 9 & 4 & 10 & 2.26 \\
\hline \multicolumn{7}{|l|}{ Mother's Education } \\
\hline At least High School & 199 & 90.5 & 168 & 75.3 & 367 & 82.84 \\
\hline College/Postgraduate & 20 & 9.1 & 51 & 22.9 & 71 & 16.03 \\
\hline Deceased/No Data & 1 & 0.4 & 4 & 1.8 & 5 & 1.13 \\
\hline \multicolumn{7}{|l|}{ Father's Education } \\
\hline At Least High School & 183 & 83.2 & 164 & 73.5 & 347 & 78.33 \\
\hline College/Postgraduate & 26 & 11.8 & 57 & 25.6 & 83 & 18.74 \\
\hline Deceased/No Data & 11 & 5 & 2 & 0.9 & 13 & 2.93 \\
\hline Average IgG, g/L & 220 & $0.046 \pm 0.036$ & 223 & $0.044 \pm 0.033$ & & \\
\hline
\end{tabular}

As shown in Figure 2, the container indices were not significantly different in control and treatment areas at baseline $\left(\mathrm{F}_{1,44}=1.43, p\right.$-value $\left.=0.239\right)$, but fractionally higher in the treatment area at month one post-treatment $\left(\mathrm{F}_{1,56}=4.18, p\right.$-value $\left.=0.046\right)$. In the control areas, the CIs increased over time. By contrast, they decreased in the treatment areas and were significantly lower by the first post-intervention sampling, two months later $\left(\mathrm{F}_{1,49}=11.64, p\right.$-value $\left.=0.001\right)$ and increasingly so in months three $\left(\mathrm{F}_{1,45}=24.15, p\right.$-value $\left.<0.001\right)$ and four $\left(\mathrm{F}_{1,42}=26.6, p\right.$-value $\left.<0.001\right)$. 


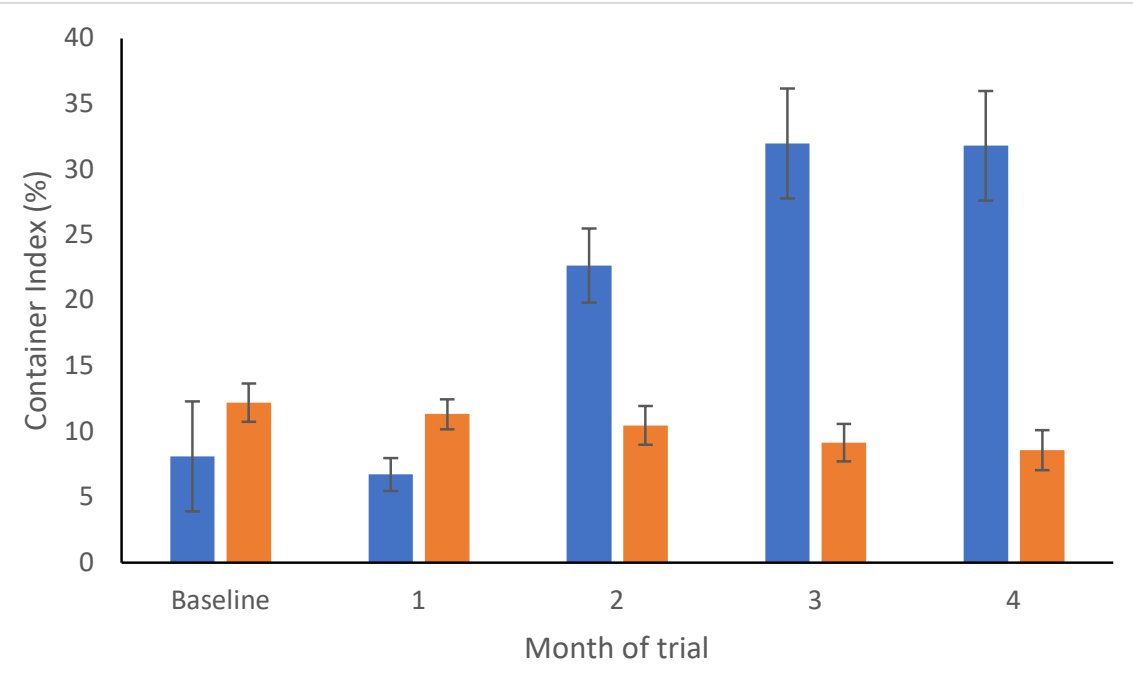

Figure 2. Container indices (Arcsine transformed to yield mean and SEM) in treatment (orange) and control (blue) areas over time.

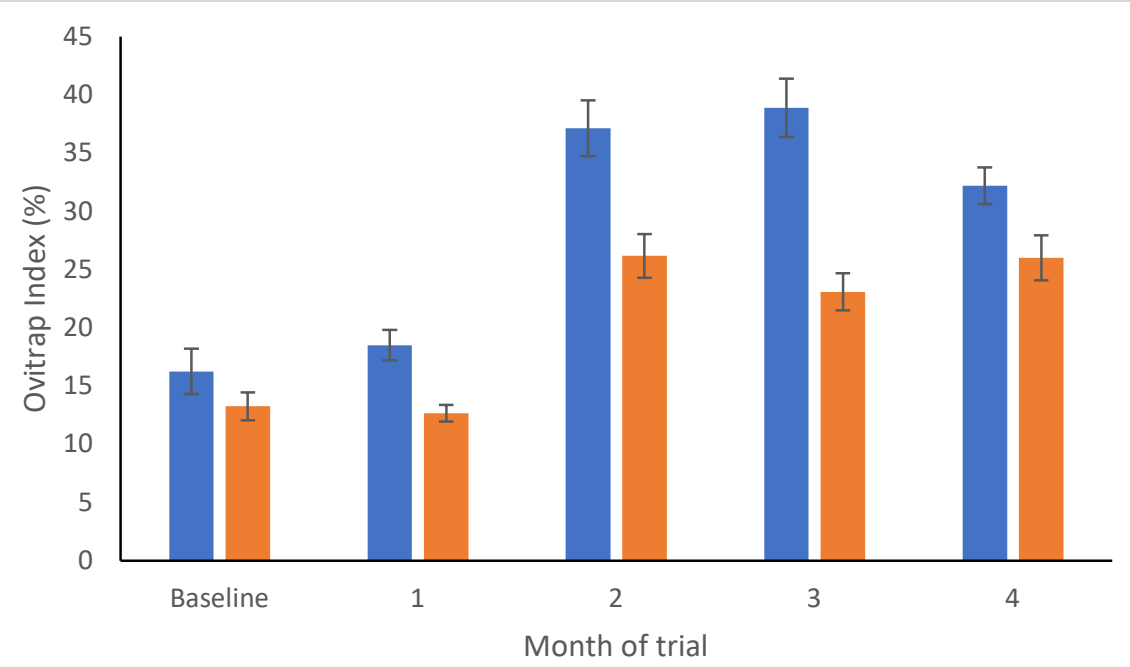

Figure 3. Ovitrap indices (Arcsine transformed to yield mean and SEM) in treatment (orange) and control (blue) areas over time.

As shown in Figure 3, at baseline the percentage of positive ovitraps was not different in the control and treatment areas $\left(\mathrm{F}_{1,89}=1.92, p\right.$-value $\left.=0.17\right)$, but started to become higher in the control areas in month $1\left(\mathrm{~F}_{1,86}=16.4, p\right.$-value $\left.<0.001\right)$. The OIs then increased significantly in both areas two months later, after which they remained relatively stable within each site type (Fig 3). However, the OI increased significantly more in the control than in the intervention sites at month $2\left(\mathrm{~F}_{1,85}=13.1, p\right.$-value $\left.<0.001\right)$ and remained as such in months three $\left(\mathrm{F}_{1,85}=28.9, p\right.$-value $\left.<0.001\right)$ and four $\left(\mathrm{F}_{1,84}=5.85, p\right.$-value $\left.=0.018\right)$.

Over the months following treatment implementation, there were twenty-two, eight, and three seroconversions at Months 2, 3, and 4 in the intervention area, respectively, and two, six, and seven in the control area for the same months of data collection (Table 2). No information was available concerning their symptomatic status potentially associated with the presumed infection. In Month 2, 16 clusters registered seroconversions in the intervention sites, one with four seropositives, three with two seropositives and 12 with 
one seropositive. In the 21 houses harboring a seroconversion in the treatment area, only one of the six houses containing more than one tested individual yielded a second seroconversion. In the control site, there were two single seropositive clusters. Of the two houses harboring a seroconversion, one contained more than one person tested, but only one seroconversion was observed. In month 3 , in the treatment site, there were two clusters with two seropositives and four clusters with single seropositives. These seroconversions occurred in seven houses; of the seven houses with seroconversions, three houses had more than one person sampled and one yielded two seroconversions. In the control site, there was one cluster with two seroconversions and four with single seropositives, with one seroconversion occurring in each of six houses. Only one of these houses had more than one person sampled. In month 4 , there were three single seroconversion clusters in the intervention site, occurring in three different houses. There were seven single seroconversion clusters in the control site. Five of the houses with a seroconversion contained more than one sampled person, but none yielded more than one seroconversion.

Table 2. Distribution of dengue seropositive individuals during the study.

\begin{tabular}{|c|c|c|c|c|}
\hline \multirow[b]{2}{*}{ Groups } & \multicolumn{4}{|c|}{ Number of dengue seropositive individuals } \\
\hline & $\begin{array}{c}\text { Baseline } \\
\text { Intervention } \\
(\mathrm{N}=220) \\
\text { Control } \\
(\mathrm{N}=223)\end{array}$ & $\begin{array}{c}\text { Month } 2 \\
\text { Intervention } \\
(\mathrm{N}=203) \\
\text { Control }(\mathrm{N}=210)\end{array}$ & $\begin{array}{c}\text { Month } 3 \\
\text { Intervention } \\
(\mathrm{N}=181) \\
\text { Control } \\
(\mathrm{N}=208)\end{array}$ & $\begin{array}{c}\text { Month } 4 \\
\text { Intervention } \\
(\mathrm{N}=173) \\
\text { Control }(\mathrm{N}=202)\end{array}$ \\
\hline Intervention & 17 & 22 & 8 & 3 \\
\hline Control & 13 & 2 & 6 & 7 \\
\hline
\end{tabular}

In a global statistical analysis across all months, there was no association of seroconversion with any of the demographic and mosquito data of the same month (Arcsin\%OI: $\chi^{2}{ }_{1}=1.89, p$-value $=0.17 ;$ Arcsin $\% C I: \chi^{2}{ }_{1}=0.45, p$-value $=0.502 ;$ Age $\chi^{2}{ }_{2}=0.47, p$-value $=0.47 ;$ Sex $\chi^{2}{ }_{1}=1.55, p$-value $=0.214$ ). Neither Arcsin\%OI nor Sex were found to be associated with seroconversion in the multivariate analysis. Lagged mosquito indices were also analyzed for any association with subsequent seroconversion rates, but none were found to be significant.

As might be expected from the large number of seroconversions in the intervention site in Month 2 (Table 2) and a subsequent decrease in the following months, there was a significant interaction effect between site type and month (Sitetype.month: $\chi^{2} 2=20.02, p$ value $<0.001)$. Following the higher $\mathrm{OR}$ of seroconversion in Month 2 in the intervention site (OR=12.16 95\%CI 3.64 - 40.59), the ORs decreased steadily in Months 3 and 4 in the intervention site (Month 3: OR=1.51 95\%CI 0.57-3.98; Month 4: OR=0.46 95\%CI 0.15-1.43). This sharp decrease from Month 2 contrasts with the control site where the ORs increased substantially in Months 3 \& 4 (Figure 4). As above, no variables were found to be associated with IgG ODs. However, a similar trend was observed when examining the changes in IgG optical densities over time (Figure 5). Treatment was not, however, observed to be significantly associated with a decrease in seroconversion or IgG optical density even at month 4 of treatment (Month 4 treatment vs. Control: Seroconversion $\mathrm{t}=1.34, p$-value $=0.09$; IgG Optical density $\mathrm{t}=1.17$, $p$-value $=0.122$ ). 


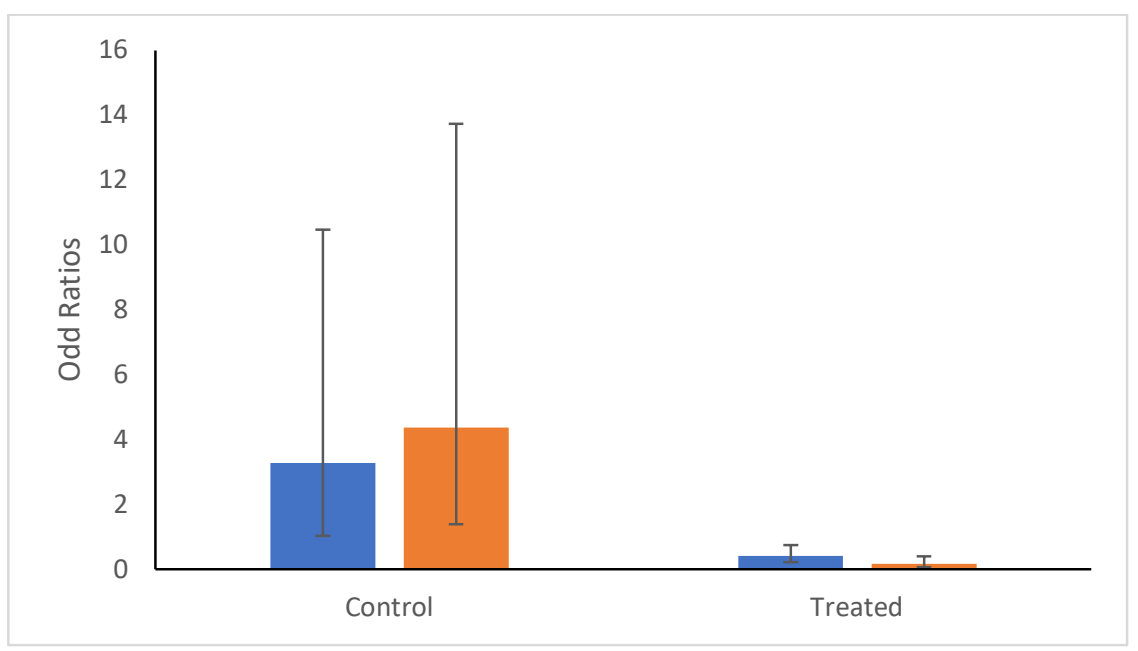

Figure 4. Odds Ratios and 95\% confidence intervals of seroconversion probabilities for Months 3 (blue) and 4 (orange) compared with Month 2 rates within each treatment type (Control or Treatment).

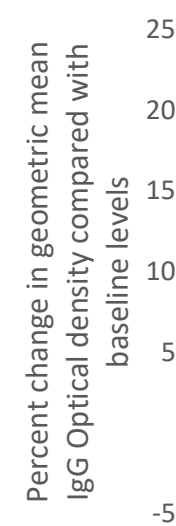

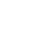

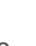

0

0

$-50$

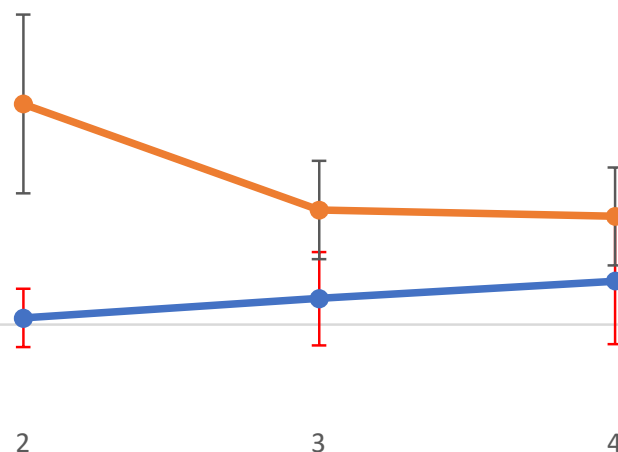

Month of Intervention

Figure 5. Percent change in geometric mean IgG optical densities as compared with baseline. Shown are means and 95\% confidence intervals for Control (blue) and Treatment (orange) areas.

\section{Discussion}

Numerous studies have assessed the entomological efficacy of application of PPF, whether through the application water sources or using an auto-dissemination approach where the mosquito would transfer small particles of PPF to other oviposition sites [2835]. With application of a granular formulation of PPF to ovitraps in study houses, we observed a decrease in ovitraps and the container index after two months of treatment. The low density of treated ovitraps (one per house) might not be expected on its own to generate such a reduction, impacting only on the eggs laid in the ovitraps. However, autodissemination of insecticide contaminated water has been shown to occur, albeit at a much lower efficacy than transfer of the powder form of PPF (41-43). In addition, females exposed to such contaminated water bodies have been shown to have subsequent reduced fertility (40). The combination of these insecticidal effects may have contributed to the decrease in mosquito indices observed. It is notable that nearly $50 \%$ of the study clusters 
did not have any water-filled containers, thus limiting the number of available and competing artificial oviposition sites. The efficacy of larvicidal traps in a community setting to reduce mosquito density, which became the basis of the integrated vector control system of the Metro Manila, has previously been observed when using Novularon [36]. Because of the relatively low flight distance of $A$ e. aegypti, especially in an urban setting, the CI will reflect the mosquito population in the immediate surrounding area of the household.

Our study is the first in the Philippines to use the serological method for dengue diagnosis by measuring IgG from the collected saliva samples. However, the measurement of IgG response levels among the participants is not very specific, cross-reacting with other flaviviruses, and it is not helpful to identify dengue serotypes. In our setting, there were no case reports of co-circulating flaviviruses. The use of saliva as an alternative to sera to detect levels of antibodies, including IgG levels, has been reported to be effective in dengue and other diseases [37-39]. A study demonstrated the efficacy of using salivary IgG levels to determine primary- and secondary-DENV infections, with high sensitivity and specificity for detecting anti-DENV IgG levels [40]. Future studies on the use of saliva to diagnose dengue infection may be an alternative to serum collection, which may be warranted when the collection of specimens is hindered by several factors, such as the age of patients or health facilities.

The current implementation of DOH strategies on the control of mosquito densities may be complemented with the use of pyriproxyfen-treated ovitraps with some modifications. However, implementation on the use of pyriproxyfen-treated ovitraps in the community should be guided by standard operating procedures being closely monitored to ensure accuracy and consistency. Numerous considerations are needed to be addressed in order to proceed on the full course of pyriproxyfen-treated ovitraps implementation including distribution schemes, ovitrap maintenance, monitoring and evaluation procedures, and plans for disposing used and unused ovitraps. The acceptance of the local communities in the implementation of new strategies should also be evaluated and considered to ensure cooperation of the public [41]. However, despite these foreseeable critical points to be considered for future scaling up and implementation of pyriproxyfen-treated ovitraps, using a small community in Taguig City, our study provided insights on its efficacy which may be used as evidence towards further studies in the Philippines.

This study has several limitations, not least the small human sample size. Such a sample size would require a powerful impact of PPF treatment to be detectable. Although the observed decrease in seroconversion rate was not significant, the trend was clear and encouraging for rolling out a larger-scale study. Secondly, local structural urban topography can significantly affect adult mosquito flight range, impeding PPF dispersal capacity and/or exposure to contaminated water bodies [42,43]. In addition, our study did not consider the heterogeneity in characteristics of the urban areas. This heterogeneity should be optimized in future research designs and be taken into account through fine-scale spatial analyses. Moreover, our study is limited to the specific doses of PPF deployed using a particular design of ovitraps. Different designs of ovitraps and dosing of PPF will undoubtedly impact efficacy $[43,44]$. Finally, our study only collected data during the cooldry season. Future studies on the effect of PPF treatment in reducing dengue infection may differ during the dry season compared to the rainy season when oviposition sites are more numerous and diverse. More extensive studies on the effectiveness of PPF, which will monitor medium to long-term effects, are warranted to confirm our findings.

\section{Conclusions}

Continuing evidence of entomological efficacy of the use of PPF provides optimism that dengue incidence might be reduced if the treatment is implemented strategically. This may complement the existing vector control strategies the local health authorities are 
currently implementing. Moreover, our results showed a need to extend the exposure to PPF and saturate all houses in a community to present a significant reduction in dengue incidence.

Author Contributions: Author Contributions: Conceptualization: A.D.L., R.E.L.P Methodology, A.D.L., R.E.L.P.,K.J.M.T., Z.J.G.R., E.S.B.; Data Collection: M.J.M.A., K.J.M.T. Formal Analysis: A.D.L., E.S.B., R.E.L.P. Investigation: A.D.L., E.S.B. Resources: A.D.L.; Data Curation, A.D.L., E.S.B., Z.J.G.R.; Writing-Original Draft Preparation: A.D.L., E.S.B., Z.J.G.R., R.E.L.P., G.J.D.A.; Writing-Review \& Editing: All authors; Visualization: A.D.L., R.E.L.P. Supervision, A.D.L.; Project Administration: K.J.M.T., M.J.M.A.; Funding Acquisition: A.D.L. All authors have read and agreed to the published version of the manuscript.

Conflicts of Interest: The authors report no conflict of interest.

Funding: The study was funded by grants from the Philippine Council for Health Research and Development - Department of Science and Technology (PCHRD-DOST).

Institutional Review Board Statement: Ethics approval of the protocol was approved by the St. Luke's Medical Center College of Medicine Research Ethics Committee (Reference No. RP 17-01).

Data sharing statement: The authors confirm that some access restrictions apply to the data. The researchers interested in using the data must obtain approval from the St. Luke's Medical Center College of Medicine Research Ethics Committee. The researchers using the data are required to follow the terms of a number of clauses designed to ensure the protection of privacy and compliance with relevant Data Privacy Act of the Philippines. Data requests may be subject to further review by the Ethics Committee and may also be subject to individual participant consent.

\section{References:}

1. WHO. Global strategy for dengue prevention and control 2012-2020. 2012.

2. Chen, L.H.; Wilson, M.E. The role of the traveler in emerging infections and magnitude of travel. Medical Clinics of North America 2008, 92, 1409-1432.

3. Christophers, S. Aedes aegypti (L.) the yellow fever mosquito: its life history, bionomics and structure. Aëdes aegypti (L.) the Yellow Fever Mosquito: its Life History, Bionomics and Structure. 1960.

4. MG, G.K.G.; Kouri, G. Dengue: an update. Lancet Infect Dis 2002, 2, 33-42.

5. Gubler, D.J. Epidemic dengue/dengue hemorrhagic fever as a public health, social and economic problem in the 21st century. Trends in microbiology 2002, 10, 100-103.

6. Rezza, G.; Nicoletti, L.; Angelini, R.; Romi, R.; Finarelli, A.; Panning, M.; Cordioli, P.; Fortuna, C.; Boros, S.; Magurano, F. Infection with chikungunya virus in Italy: an outbreak in a temperate region. The Lancet 2007, 370 , 1840-1846.

7. Radke, E.G.; Gregory, C.J.; Kintziger, K.W.; Sauber-Schatz, E.K.; Hunsperger, E.A.; Gallagher, G.R.; Barber, J.M.; Biggerstaff, B.J.; Stanek, D.R.; Tomashek, K.M. Dengue outbreak in key west, Florida, USA, 2009. Emerging infectious diseases 2012, 18, 135.

8. Sousa, C.; Clairouin, M.; Seixas, G.; Viveiros, B.; Novo, M.; Silva, A.; Escoval, M.; Economopoulou, A. Ongoing outbreak of dengue type 1 in the Autonomous Region of Madeira, Portugal: preliminary report. Eurosurveillance 2012, 17, 20333. 
9. Murray, K.O.; Rodriguez, L.F.; Herrington, E.; Kharat, V.; Vasilakis, N.; Walker, C.; Turner, C.; Khuwaja, S.; Arafat, R.; Weaver, S.C. Identification of dengue fever cases in Houston, Texas, with evidence of autochthonous transmission between 2003 and 2005. Vector-Borne and Zoonotic Diseases 2013, 13, 835-845.

10. Paul, R.E. The when and the where of Zika epidemic potential in Europe-an evidence base for public health preparedness. EBioMedicine 2016, 9, 17-18.

11. Giron, S.; Franke, F.; Decoppet, A.; Cadiou, B.; Travaglini, T.; Thirion, L.; Durand, G.; Jeannin, C.; L'ambert, G.; Grard, G. Vector-borne transmission of Zika virus in Europe, southern France, August 2019. Eurosurveillance 2019, 24, 1900655.

12. Faulde, M.K.; Rueda, L.M.; Khaireh, B.A. First record of the Asian malaria vector Anopheles stephensi and its possible role in the resurgence of malaria in Djibouti, Horn of Africa. Acta tropica 2014, 139, 39-43.

13. Sinka, M.; Pironon, S.; Massey, N.; Longbottom, J.; Hemingway, J.; Moyes, C.; Willis, K. A new malaria vector in Africa: Predicting the expansion range of Anopheles stephensi and identifying the urban populations at risk. Proceedings of the National Academy of Sciences 2020, 117, 24900-24908.

14. Ligsay, A.; Telle, O.; Paul, R. Challenges to Mitigating the Urban Health Burden of Mosquito-Borne Diseases in the Face of Climate Change. International Journal of Environmental Research and Public Health 2021, 18, 5035.

15. Nash, D.; Mostashari, F.; Fine, A.; Miller, J.; O'leary, D.; Murray, K.; Huang, A.; Rosenberg, A.; Greenberg, A.; Sherman, M. The outbreak of West Nile virus infection in the New York City area in 1999. New England Journal of Medicine 2001, 344, 1807-1814.

16. Huhn, G.D.; Austin, C.; Langkop, C.; Kelly, K.; Lucht, R.; Lampman, R.; Novak, R.; Haramis, L.; Boker, R.; Smith, S. The emergence of West Nile virus during a large outbreak in Illinois in 2002. The American journal of tropical medicine and hygiene 2005, 72, 768-776.

17. Ruiz, M.O.; Walker, E.D.; Foster, E.S.; Haramis, L.D.; Kitron, U.D. Association of West Nile virus illness and urban landscapes in Chicago and Detroit. International Journal of Health Geographics 2007, 6, 1-11.

18. Murray, N.E.A.; Quam, M.B.; Wilder-Smith, A. Epidemiology of dengue: past, present and future prospects. Clinical epidemiology 2013, 5, 299.

19. Ebi, K.L.; Nealon, J. Dengue in a changing climate. Environmental research 2016, 151, 115-123.

20. Bowman, L.R.; Donegan, S.; McCall, P.J. Is dengue vector control deficient in effectiveness or evidence?: Systematic review and meta-analysis. PLoS neglected tropical diseases 2016, 10, e0004551.

21. Bartlett-Healy, K.; Hamilton, G.; Healy, S.; Crepeau, T.; Unlu, I.; Farajollahi, A.; Fonseca, D.; Gaugler, R.; Clark, G.G.; Strickman, D. Source reduction behavior as an independent measurement of the impact of a public health education campaign in an integrated vector management program for the Asian tiger mosquito. International journal of environmental research and public health 2011, 8, 1358-1367.

22. WHO-UNICEF. Global vector control response 2017-2030. 2017.

23. WHO. Dengue: guidelines for diagnosis, treatment, prevention and control; World Health Organization: 2009.

24. Andries, A.-C.; Duong, V.; Ong, S.; Ros, S.; Sakuntabhai, A.; Horwood, P.; Dussart, P.; Buchy, P. Evaluation of the performances of six commercial kits designed for dengue NS1 and anti-dengue IgM, IgG and IgA detection in urine and saliva clinical specimens. BMC infectious diseases 2016, 16, 1-9.

25. Andries, A.-C.; Duong, V.; Ly, S.; Cappelle, J.; Kim, K.S.; Lorn Try, P.; Ros, S.; Ong, S.; Huy, R.; Horwood, P. Value of routine dengue diagnostic tests in urine and saliva specimens. PLoS neglected tropical diseases 2015, 9, e0004100.

26. Hart, G.F. Transformation of variables for compositional data-analysis. 2011. 
27. Loomis, D.; Richardson, D.B.; Elliott, L. Poisson regression analysis of ungrouped data. Occupational and environmental medicine 2005, 62, 325-329.

28. Itoh, T.; Kawada, H.; Abe, A.; Eshita, Y.; Rongsriyam, Y.; Igarashi, A. Utilization of bloodfed females of Aedes aegypti as a vehicle for the transfer of the insect growth regulator pyriproxyfen to larval habitats. Journal of the American Mosquito Control Association-Mosquito News 1994, 10, 344-347.

29. Devine, G.J.; Overgaard, H.J.; Paul, R.E. Global vector control guidelines-the need for co-creation. Trends in parasitology 2019, 35, 267-270.

30. Abad-Franch, F.; Zamora-Perea, E.; Luz, S.L. Mosquito-disseminated insecticide for citywide vector control and its potential to block arbovirus epidemics: entomological observations and modeling results from Amazonian Brazil. PLoS medicine 2017, 14, e1002213.

31. Ohba, S.-y.; Ohashi, K.; Pujiyati, E.; Higa, Y.; Kawada, H.; Mito, N.; Takagi, M. The effect of pyriproxyfen as a "population growth regulator" against Aedes albopictus under semi-field conditions. PLoS One 2013, 8, e67045.

32. Unlu, I.; Suman, D.S.; Wang, Y.; Klingler, K.; Faraji, A.; Gaugler, R. Effectiveness of autodissemination stations containing pyriproxyfen in reducing immature populations Aedes albopictus. Parasites E Vectors $2017,10$.

33. Seixas, G.; Paul, R.E.; Pires, B.; Alves, G.; de Jesus, A.; Silva, A.-C.; Devine, G.J.; Sousa, C.A. An evaluation of efficacy of the auto-dissemination technique as a tool for Aedes aegypti control in Madeira, Portugal. Parasites $\mathcal{E}$ vectors 2019, 12, 1-13.

34. Ritchie, S.A.; Paton, C.; Buhagiar, T.; Webb, G.A.; Jovic, V. Residual treatment of Aedes aegypti (Diptera: Culicidae) in containers using pyriproxyfen slow-release granules (Sumilarv 0.5 G). Journal of medical entomology 2013, 50, 1169-1172.

35. Sihuincha, M.; Zamora-Perea, E.; Orellana-Rios, W.; Stancil, J.D.; López-Sifuentes, V.; Vidal-Ore, C.; Devine, G.J. Potential use of pyriproxyfen for control of Aedes aegypti (Diptera: Culicidae) in Iquitos, Peru. Journal of medical entomology 2005, 42, 620-630.

36. De Las Llagas, L.A.; Bersales, L.G.S.; Bertuso, A.G.; Mistica, M.S.; Ocampo, V.R.; Samaniego, J.B.; Magsino, E.A.; Alzona, F.D. Mosquito Larvicidal Trap (MLT) as surveillance and control tool for Aedes mosquitoes. Southeast Asian Journal of Tropical Medicine and Public Health 2016, 47, 701.

37. da Silva, M.V.; Camargo, E.D.; Vaz, A.J.; Batista, L. Immunodiagnosis of human leptospirosis using saliva. Transactions of the Royal Society of Tropical Medicine and Hygiene 1992, 86, 560-561.

38. Frerichs, R. Saliva assays for HIV antibody diagnosis. Labmed. Int 1994, 5, 16-19.

39. Friedman, M.G.; Phillip, M.; Dagan, R. Virus-specific IgA in serum, saliva, and tears of children with measles. Clinical and experimental immunology 1989, 75, 58.

40. Cuzzubbo, A.J.; Vaughn, D.W.; Nisalak, A.; Suntayakorn, S.; Aaskov, J.; Devine, P.L. Detection of specific antibodies in saliva during dengue infection. Journal of clinical microbiology 1998, 36, 3737-3739.

41. Organization, W.H. Efficacy-testing of traps for control of Aedes spp. mosquito vectors; World Health Organization: 2018.

42. WHO. Guidelines for diagnosis, Treatment. Prevention and Control 2009, 1.

43. Wang, Y.; Suman, D.S.; Bertrand, J.; Dong, L.; Gaugler, R. Dual - treatment autodissemination station with enhanced transfer of an insect growth regulator to mosquito oviposition sites. Pest management science 2014, 70, 1299-1304.

44. Iwanaga, K.; Kanda, T. The effects of a juvenile hormone active oxime ether compound on the metamorphosis and reproduction of an anopheline vector, Anopheles balabacensis (Diptera: Culicidae). Applied Entomology and Zoology 1988, 23, 186-193. 
